\title{
Fatores associados ao excesso de peso entre manipuladores de alimentos de escolas públicas"
} Factors associated with overweight among food handlers in public schools

\author{
Thamiris Rodrigues Macedo* \\ Adriana Fernandez Versiani dos Anjos** \\ Luana Caroline dos Santos*** \\ Maria Flávia Gazzinelli Bethony *** \\ Simone Cardoso Lisboa Pereira*****
}

\begin{abstract}
Resumo
O estado nutricional é um dos indicadores do comportamento e hábito alimentar e a maneira como a pessoa se alimenta está envolvida em muitos significados, podendo influenciar no modo como ela prepara as refeições. Nesse sentido, destaca-se o manipulador de alimentos no processo de formação de comportamentos e atitudes relativos às escolhas alimentares e à convivência social dos escolares. Portanto, o objetivo deste estudo foi caracterizar o estado nutricional e identificar os fatores associados ao excesso de peso entre manipuladores de alimentos de escolas públicas, subsídeo para o planejamento de ações de promoção de saúde nas instituições e valorização dos manipuladores de alimentos neste processo. Estudo analítico conduzido com manipuladores de alimentos de escolas públicas municipais de Belo Horizonte, Minas Gerais , em 2013. O excesso de peso foi diagnosticado pelo índice de massa corporal e associado com variáveis antropométricas, socioeconômicas, história de saúde e hábitos alimentares, obtidas por meio de questionário semiestruturado. Participaram 274 sujeitos, 99,6\% do sexo feminino, maioria casada, adulta, com ensino fundamental e renda per capita de até um salário mínimo. A prevalência de excesso de peso foi de $82,80 \%$ e se associou positivamente com a presença de hipercolesterolemia, hipertrigliceridemia, uso de medicamentos, hábito de beliscar; e inversamente com o consumo adequado de água e a prática de atividade física. A elevada prevalência de excesso de peso e seus fatores associados sinalizam a necessidade de planejamento e implementação de ações de educação em saúde para a prevenção e controle desse agravo entre os manipuladores de alimentos.
\end{abstract}

Palavras-chave: Manipuladores de Alimentos. Alimentação Escolar. Estado Nutricional. Excesso de Peso. Hábitos Alimentares.

\begin{abstract}
Nutritional status is one of the indicators of behavior and food habits, and the way a person feeds is involved in many meanings and can influence the way she prepares meals. In this sense, food handlers are very important in the development of attitudes and behaviors relating to food choices and social life in the school setting. Therefore, the aim of this study was to characterize the nutritional status and identify the factors associated with excess weight of food handlers in public schools. This is an analytical study conducted with food handlers in public schools in Belo Horizonte, Minas Gerais, in 2013. Overweight was diagnosed by means of body mass index measurements and was correlated to anthropometric, socioeconomic, health history and food habit variables that were obtained through a semi-structured questionnaire. 274 subjects participated, among whom $99.6 \%$ female, mostly married, adult with primary education and per capita income up to the Brazilian national minimum wage were studied. The prevalence of overweight was $82.80 \%$, positively associated with hypercholesterolemia, hypertriglyceridemia, use of medications, habit of snacking, and inversely with the recommended water intake and physical activity practice. The high prevalence of overweight and some factors associated signal the need for planning and implementing actions of health education for the prevention and control of this condition among food handlers in public schools.
\end{abstract}

Keywords: Food Handlers. School Feeding. Nutritional Status. Overweight. Food Habits.

DOI: 10.15343/0104-7809.20153902210218

\# Pesquisa financiada pela Fundação de Amparo à Pesquisa do Estado de Minas Gerais - FAPEMIG, Processo APQ-02812-13.

* Universidade Federal de Minas Gerais. Belo Horizonte, MG - Brasil. E-mail: thamirisrm@yahoo.com.br.

** Prefeitura Municipal de Belo Horizonte. Belo Horizonte, MG - Brasil. E-mail: driarroba@gmail.com.

*** Universidade Federal de Minas Gerais. Belo Horizonte, MG - Brasil. E-mail: luanacs@ig.com.br.

**** Universidade Federal de Minas Gerais, Belo Horizonte, MG - Brasil. E-mail: flaviagazzinelli@yahoo.com.br.

***** Universidade Federal de Minas Gerais, Belo Horizonte - Minas Gerais - Brasil, E-mail: simoneclpereira@gmail.com.

Os autores declaram não haver conflito de interesses. 


\section{INTRODUÇÃO}

O manipulador de alimento, na perspectiva de uma terminologia adotada internacionalmente, refere-se ao profissional que prepara, distribui e, ou comercializa os alimentos ${ }^{1-2}$. Desse se almeja o cumprimento das boas práticas na manipulação e preparo dos alimentos a fim de se garantir e promover a segurança alimentar ${ }^{3}$. Destaca-se que, embora o aparato tecnológico disponível para a produção de alimentos seja significativo, a qualidade sensorial e nutricional das refeições ainda está diretamente relacionada ao desempenho desses profissionais ${ }^{4}$.

No âmbito do Programa Nacional de Alimentação Escolar - PNAE, o manipulador de alimentos adquire especial relevância pelo seu papel. No processo de formação de comportamentos e atitudes relativos às escolhas alimentares e à convivência social dos escolares ${ }^{5}$. No entanto, sabe-se que a maneira como a pessoa se alimenta está envolvida em muitos significados, podendo influenciar o modo como prepara as refeições ${ }^{6}$.

Um dos indicadores do comportamento e hábito alimentar é o estado nutricional e estudos revelam alto índice de excesso de peso entre os manipuladores de alimentos, sugerindo a contribuição da atividade profissional para tal quadro, bem como a interferência negativa do referido desvio nutricional para a atividade, tornando-a mais desgastante ${ }^{7-8}$.

Diante deste cenário e escassez de estudos sobre esta importante temática, o presente estudo tem por objetivo caracterizar o estado nutricional dos manipuladores de alimentos de escolas públicas municipais e identificar os fatores socioeconômicos, de saúde e nutricional, associados ao excesso de peso nesta população.

\section{MÉTODOS}

Trata-se de um estudo transversal descritivo analítico, realizado com manipuladores de alimentos de escolas públicas municipais de Belo Horizonte, Minas Gerais. Os participantes receberam informações sobre o estudo e assinaram um termo de consentimento livre e esclarecido. O projeto, qual este estudo compõe, foi aprovado pelo Comitê de Ética em Pesquisa/COEP da Universidade Federal de Minas Gerais (UFMG), protocolo n ${ }^{\circ} 00734412.0 .0000 .5149$.

O cálculo amostral foi efetuado a partir das fórmulas para fins descritivos propostas por Browner et al (2003) ${ }^{9}$. Para tal, adotou-se 95\% de intervalo de confiança, erro amostral máximo de $10 \%$ e as variabilidades proporcionais dos manipuladores de alimentos, por distrito sanitário, obtidas por meio das informações da Prefeitura Municipal. Foi estimulada a participação de 248 manipuladores de alimentos representantes das escolas, sorteadas aleatoriamente, distribuídas nos nove distritos sanitários de Belo Horizonte, no ano de 2013.

A coleta de dados constou de aferições antropométricas e aplicação face a face de um questionário semiestruturado.

As medidas antropométricas massa corporal, altura, perímetro de cintura e de quadril, foram aferidas por meio de balança digital Plenna ${ }^{\circledR}$ com capacidade para $150 \mathrm{~kg}$ e precisão de 100 g; estadiômetro Alturexata ${ }^{\circledR}$ com intervalo 0 a $0,80 \mathrm{~m}$ e precisão de $1 \mathrm{~mm}$; e fita métrica inelástica, respectivamente. Estas medidas foram aferidas três vezes, calculando-se a média aritmética individual.

A partir dos dados de peso e altura, a avaliação nutricional foi realizada calculando-se o índice de massa corporal (IMC=peso/altura ${ }^{2}$ ), que foi classificado de modo diferenciado para adultos $^{10}$ e idosos $(\geq 60 \text { anos de idade })^{11}$, devido as diferenças fisiológicas de cada faixa etária. As categorias de sobrepeso e obesidade foram agrupadas para configurar o excesso de peso.

Os dados de perímetro de cintura possibilitaram classificar os manipuladores de alimentos quanto ao risco de desenvolver complicações metabólicas associadas à obesidade e em conjunto com os dados de perímetro de quadril expressaram a relação cintura quadril que determina o risco para o desenvolvimento de doenças ${ }^{10}$.

O questionário aplicado, por equipe devidamente treinada, visou coletar informações socioeconômicas (renda familiar, estado civil, escolaridade, idade), história de saúde (morbidades referidas: diabetes, hipertensão arterial,
211 
colesterol e triglicérides elevados, diarreia e constipação; uso de medicamentos; tabagismo; sono; e prática de atividade física) e hábitos alimentares (consumo de frutas, óleo, sal, açúcar e água; hábito de beliscar e de realizar as refeições em frente à televisão) dos sujeitos do estudo ${ }^{12}$.

Para a determinação da categorização de renda per capita os indivíduos foram divididos mínimo vigente em 2013(R $\$ 678,00)$, de $1 / 4$ a $1 / 2$, de $1 / 2$ a 1 , de 1 a 2,2 a 5 e 5 ou mais salários ${ }^{13}$.

Quanto à prática de atividade física, os manipuladores de alimentos foram classificados em inativos ( $<3$ horas/semana) e ativos a partir dos critérios do Institute of Medicine (2006) ${ }^{15}$.

Em relação aos hábitos alimentares, as variáveis foram categorizadas conforme a recomendação de consumo diário do Guia Alimentar para a População Brasileira ${ }^{11}$ : consumo per capita adequado de óleo - menor ou igual a $8 \mathrm{~mL}$; sal - menor ou igual a 5 gramas; açúcar - menor ou igual a 28 gramas; frutas - 3 porções; e água - 2 litros $^{16}$.

Os dados obtidos foram analisados com auxílio do software Statistical Package for the Social Science (SPSS), versão 17.0 for Windows ${ }^{\circledR}$. Realizou-se análise descritiva e aplicação dos testes Kolmogorov-Smirnov (avaliar a aderência das variáveis quantitativas à distribuição normal) e qui-quadrado (estudar a associação de excesso de peso com as demais variáveis).

As variáveis com $p<0,25$ na análise univariada foram selecionadas para a análise multivariada por meio da regressão de Poisson com variâncias robustas. Calcularam-se as razões de prevalência ajustadas e os respectivos intervalos de confiança de $95 \%$. Permaneceram no modelo final as variáveis significativas ao nível de $5 \%$, adotou-se critério de eliminação Backward. $\mathrm{O}$ ajuste final do modelo foi realizado por meio do Teste Hosmer e Lemeshow. Tais análises foram realizadas no software Stata (Statistical Analysis System Software) versão 11.2, adotando-se nível de significância de 5\% $(p<0,05)$.

As variáveis paramétricas foram apresentadas na forma de média e desvio-padrão, enquanto as demais estarão descritas como mediana e valores mínimo e máximo.

\section{RESULTADOS}

Participaram deste estudo 274 manipuladores de alimentos, seguindo a proporcionalidade dos distritos sanitários do município de Belo Horizonte, em 2013, estando mais de 80,0\% acima do peso e a maioria com risco para o desenvolvimento de doenças cardiovasculares e fatores de risco para o desenvolvimento de doenças associadas à obesidade, considerando a avaliação do perímetro da cintura e a relação cintura e quadril, respectivamente (Tabela 1).

Com relação aos aspectos demográficos e socioeconômicos pode-se observar que, na população estudada, predominaram indivíduos adultos de maior idade (idade mediana de 46 anos, IC95\%; 22-67), do sexo feminino, casados, com ensino fundamental e renda per capita de meio a um salário mínimo (Tabela 1).

No que se refere aos dados de saúde, pode-se observar que os manipuladores de alimentos declararam apresentar diabetes, hipertensão arterial, colesterol elevado, triglicérides elevadas e utilizam medicamentos; com maior prevalência de hipertensão (35,4\%). Além disso, cerca de $25 \%$ alegam não dormir bem e a maioria (59,8\%) não pratica atividade física.

Ao analisar os hábitos alimentares dos manipuladores de alimentos, constatou-se que a maioria não apresenta um consumo adequado de frutas, mantém um consumo excessivo de óleo e açúcar, ingere água em quantidade insuficiente e adota a prática de beliscar alimentos entre as refeições.

Na análise univariada, o excesso de peso foi mais prevalente entre os participantes que referiram apresentar hipertensão arterial $(91,80 \%$ vs $77,60 \% ; p=0,002)$, colesterol elevado $(100,00 \%$ vs $79,20 \% ; p=0,001)$ e uso de medicamentos $(90,40 \%$ vs $76,50 \%$; $p=0,002)$, além daqueles com hábito de beliscar $(88,20 \%$ vs $75,20 \%$; $p=0,006)$, não tabagistas $(70,70 \%$ vs $85,00 \% ; p=0,041)$ e praticantes de atividade física $(95,50 \%$ vs 78,80\%; $p=0,001)$ (Tabela 2). Nenhum fator socioeconômico se associou significativamente ao excesso de peso. 
Tabela 1. Estado nutricional E Perfil socioeconômico de manipuladores de alimentos das escolas municipais de Belo Horizonte - MG, 2013. ( $n=274$ )

\begin{tabular}{|c|c|c|}
\hline ANTROPOMETRIA & $\mathbf{n}$ & $\%$ \\
\hline \multicolumn{3}{|l|}{ Classificação do Índice de Massa Corporal } \\
\hline Baixo Peso & 3 & 1,1 \\
\hline Eutrofia & 44 & 16,1 \\
\hline Excesso de peso & 227 & 82,8 \\
\hline Obesidade (mulheres adultas, $n=265$ ) & 126 & 47,55 \\
\hline Sobrepeso & 101 & 36,86 \\
\hline \multicolumn{3}{|l|}{ Classificação do Perimetro de Cintura } \\
\hline Sem Risco & 54 & 19,7 \\
\hline Elevado & 72 & 26,3 \\
\hline Muito Elevado & 148 & 54 \\
\hline \multicolumn{3}{|l|}{ Classificação da Relação Cintura Quadril } \\
\hline Sem Risco & 107 & 39,1 \\
\hline $\begin{array}{l}\text { Risco para desenvolvimento de } \\
\text { doenças }\end{array}$ & 167 & 60,9 \\
\hline CARACTERÍSTICAS & $\mathbf{n}$ & $\%$ \\
\hline \multicolumn{3}{|l|}{ Idade } \\
\hline Adulto (20 - 59 anos) & 264 & 96,4 \\
\hline Idosos (60 anos ou mais) & 10 & 3,6 \\
\hline \multicolumn{3}{|l|}{ Sexo } \\
\hline Feminino & 273 & 99,6 \\
\hline Masculino & 1 & 0,4 \\
\hline \multicolumn{3}{|l|}{ Estado Civil } \\
\hline Solteiro & 57 & 20,8 \\
\hline Casado & 168 & 61,3 \\
\hline Divorciado & 40 & 14,6 \\
\hline Viúvo & 9 & 3,3 \\
\hline \multicolumn{3}{|l|}{ Escolaridade } \\
\hline Nunca estudou & 6 & 2,2 \\
\hline Ensino Fundamental Incompleto & 41 & 15 \\
\hline Ensino Fundamental & 122 & 44,5 \\
\hline Ensino Médio & 98 & 35,7 \\
\hline Ensino Superior & 7 & 2,6 \\
\hline \multicolumn{3}{|l|}{ Renda Per capita * } \\
\hline Até 1/4 SM & 24 & 8,7 \\
\hline $1 / 4$ a $1 / 2$ SM & 74 & 27 \\
\hline 1/2 a 1 SM & 129 & 47,1 \\
\hline 1 a 2 SM & 42 & 15,3 \\
\hline 2 a 5 SM & 4 & 1,5 \\
\hline 5 ou Mais SM & 1 & 0,4 \\
\hline
\end{tabular}

* SM: Salário mínimo.
Na análise multivariada, a prevalência de excesso de peso manteve-se maior entre os manipuladores com hipercolesterolemia ( $R P=1,14$, IC95\%:1,04-1,26), uso de medicamentos $(\mathrm{RP}=1,19$, IC95\%:1,05-1,34) e o hábito de beliscar alimentos (RP=1,15, IC95\%:1,01-1,30), além daqueles hipertrigliceridemia $(R P=1,11$; IC 95\%: 1,00-1,23). Em contraste, a prevalência foi menor entre os participantes inativos fisicamente $(\mathrm{RP}=0,81$; IC95\%: 0,73-0,89) e que declararam não consumir a quantidade recomendada de água ( $R P=0,88$; IC95\%: 0,79-0,99) (Tabela 3).

\section{DISCUSSÃO}

A relevância deste estudo está pautada no fato de que as informações relativas à prevalência de excesso de peso, bem como os fatores relacionados ao perfil levantado, em meio à população estudada podem sinalizar para a necessidade premente de ações de promoção de práticas alimentares saudáveis voltadas a este público. Adicionalmente, apontam-se aspectos interessantes para estudos qualitativos futuros, pautados em representações sociais. Isto, pois se acredita que a maneira como a pessoa se alimenta está envolvida em muitos significados, que podem influenciar no seu papel enquanto promotor da alimentação saudável.

Ao analisar o estado nutricional, verificou-se que a prevalência de excesso de peso ora detectada evidencia os níveis epidêmicos deste problema, sendo ainda superior aos achados às prevalências de outros estudos com populações semelhantes. Em manipuladores de alimentos do serviço de nutrição e dietética de oito hospitais públicos estaduais da cidade de Florianópolis detectou-se $71,5 \%$ de excesso de peso ${ }^{17}$. Similarmente, numa empresa localizada no sul do Brasil, 71,4\% dos manipuladores de alimentos apresentaram excesso de peso ${ }^{7}$. Ainda no Sul do país, Scarparo e colaboradores identificaram $56,9 \%$ de excesso de peso entre manipuladores de alimentos de restaurantes universitários ${ }^{8}$. Ressalta-se que não foram localizados estudos publicados, nacionais e, ou internacionais, avaliando o estado nutricional de manipuladores de alimentos de escolas públicas e, ou privadas, para comparações. 
Tabela 2. Prevalência de excesso de peso segundo variáveis socioeconômicas, história de saúde e hábitos alimentares em manipuladores de alimentos de escolas municipais de Belo Horizonte - MG, 2013.

\begin{tabular}{|c|c|c|c|c|c|c|}
\hline \multirow[b]{5}{*}{214} & \multirow{3}{*}{ VARIÁVEIS } & \multicolumn{5}{|c|}{ EXCESSO DE PESO } \\
\hline & & \multicolumn{2}{|c|}{ Sim } & \multicolumn{2}{|c|}{ Não } & \multirow[t]{2}{*}{$\mathbf{p}$} \\
\hline & & $\mathbf{n}$ & $\%$ & $\mathbf{n}$ & $\%$ & \\
\hline & \multicolumn{6}{|l|}{ Idade } \\
\hline & Adulto (19 - 59 anos) & 220 & 83,30 & 44 & 16,70 & \multirow{2}{*}{0,236} \\
\hline \multirow{34}{*}{ 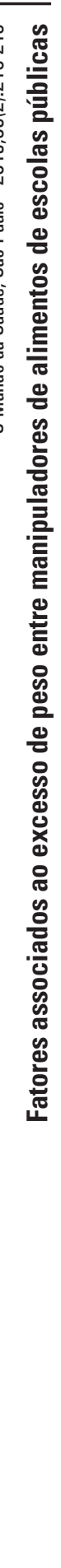 } & Idosos (60 anos ou mais) & 7 & 70,00 & 3 & 30,00 & \\
\hline & Sexo & & & & & \\
\hline & Feminino & 226 & 82,80 & 47 & 17,20 & \multirow{2}{*}{1,000} \\
\hline & Masculino & 1 & 100,00 & 0 & 0,00 & \\
\hline & \multicolumn{6}{|l|}{ Estado Civil } \\
\hline & Solteiro & 46 & 80,70 & 11 & 19,30 & \multirow{4}{*}{0,908} \\
\hline & Casado & 139 & 82,70 & 29 & 17,30 & \\
\hline & Divorciado & 34 & 85,00 & 6 & 15,00 & \\
\hline & Viúvo & 8 & 88,90 & 1 & 11,10 & \\
\hline & \multicolumn{6}{|l|}{ Escolaridade } \\
\hline & Nunca estudou & 5 & 83,30 & 1 & 16,70 & \multirow{5}{*}{0,907} \\
\hline & Ensino Fundamental Incompleto & 33 & 80,50 & 8 & 19,50 & \\
\hline & Ensino Fundamental & 103 & 84,40 & 19 & 15,60 & \\
\hline & Ensino Médio & 81 & 82,70 & 17 & 17,30 & \\
\hline & Ensino Superior & 5 & 71,40 & 2 & 28,60 & \\
\hline & \multicolumn{6}{|l|}{ Renda per capita* } \\
\hline & Até 1/4 SM & 17 & 70,80 & 7 & 29,20 & \multirow{6}{*}{0,101} \\
\hline & $1 / 4$ a $1 / 2 \mathrm{SM}$ & 62 & 83,90 & 12 & 16,20 & \\
\hline & $1 / 2$ a $1 \mathrm{SM}$ & 107 & 82,90 & 22 & 17,10 & \\
\hline & 1 a 2 SM & 38 & 90,50 & 4 & 9,50 & \\
\hline & 2 a $5 \mathrm{SM}$ & 3 & 75,00 & 1 & 25,00 & \\
\hline & 5 ou Mais SM & 0 & 0,00 & 1 & 100,00 & \\
\hline & \multicolumn{6}{|l|}{ Diabetes } \\
\hline & Sim & 18 & 94,70 & 1 & 5,30 & \multirow{2}{*}{0,131} \\
\hline & Não & 201 & 82,00 & 44 & 18,00 & \\
\hline & \multicolumn{6}{|l|}{ Hipertensão arterial } \\
\hline & Sim & 89 & 91,80 & 8 & 8,20 & \multirow{2}{*}{0,002} \\
\hline & Não & 135 & 77,60 & 39 & 22,40 & \\
\hline & \multicolumn{6}{|l|}{ Colesterol alto } \\
\hline & Sim & 33 & 100,00 & 0 & 0,00 & \multirow{2}{*}{0,001} \\
\hline & Não & 171 & 79,20 & 45 & 20,80 & \\
\hline & \multicolumn{6}{|l|}{ Triglicérides Alto } \\
\hline & Sim & 14 & 100,00 & 0 & 0,00 & \multirow{2}{*}{0,078} \\
\hline & Não & 183 & 79,90 & 46 & 20,10 & \\
\hline
\end{tabular}


...continuação - Tabela 2

\begin{tabular}{|c|c|c|c|c|c|}
\hline \multirow{3}{*}{ VARIÁVEIS } & \multicolumn{5}{|c|}{ EXCESSO DE PESO } \\
\hline & \multicolumn{2}{|c|}{ Sim } & \multicolumn{2}{|c|}{ Não } & \multirow[t]{2}{*}{$\mathbf{p}$} \\
\hline & $\mathbf{n}$ & $\%$ & $\mathbf{n}$ & $\%$ & \\
\hline \multicolumn{6}{|l|}{ Constipação } \\
\hline Sim & 39 & 86,70 & 6 & 13,30 & \multirow{2}{*}{0,524} \\
\hline Não & 188 & 82,10 & 41 & 17,90 & \\
\hline \multicolumn{6}{|l|}{ Diarreia } \\
\hline Sim & 2 & 100,00 & 0 & 0,00 & \multirow{2}{*}{1,000} \\
\hline Não & 224 & 82,70 & 47 & 17,30 & \\
\hline \multicolumn{6}{|l|}{ Medicamentos } \\
\hline Sim & 113 & 90,40 & 12 & 9,60 & \multirow{2}{*}{0,002} \\
\hline Não & 114 & 76,50 & 35 & 23,50 & \\
\hline \multicolumn{6}{|l|}{ Tabagismo } \\
\hline Sim & 29 & 70,70 & 12 & 29,30 & \multirow{2}{*}{0,041} \\
\hline Não & 198 & 85,00 & 35 & 15,00 & \\
\hline \multicolumn{6}{|l|}{ Prática de atividade física } \\
\hline Sim & 63 & 95,50 & 3 & 4,50 & \multirow{2}{*}{0,001} \\
\hline Não & 164 & 78,80 & 44 & 21,20 & \\
\hline \multicolumn{6}{|l|}{ Dorme bem } \\
\hline Sim & 165 & 82,90 & 34 & 17,10 & \multirow{2}{*}{1,000} \\
\hline Não & 62 & 82,70 & 13 & 17,30 & \\
\hline \multicolumn{6}{|l|}{ Consumo de água } \\
\hline Menos de 2 litros & 144 & 79,60 & 37 & 20,40 & \multirow{2}{*}{0,062} \\
\hline 2 litros ou mais & 83 & 89,20 & 10 & 10,80 & \\
\hline \multicolumn{6}{|l|}{ Hábito de comer assistindo TV } \\
\hline Sim & 115 & 86,50 & 18 & 13,50 & \multirow{2}{*}{0,149} \\
\hline Não & 112 & 79,40 & 29 & 20,60 & \\
\hline \multicolumn{6}{|l|}{ Hábito de beliscar } \\
\hline Sim & 142 & 88,20 & 19 & 11,80 & \multirow{2}{*}{0,006} \\
\hline Não & 85 & 75,20 & 28 & 24,80 & \\
\hline \multicolumn{6}{|l|}{ Adequação do consumo de frutas } \\
\hline Adequado (3 porções diárias ou mais) & 24 & 82,80 & 5 & 17,20 & \multirow{2}{*}{1,000} \\
\hline Inadequado (< 3 porções diárias) & 203 & 82,90 & 42 & 17,10 & \\
\hline Consumo per capita de óleo & & & & & \\
\hline Adequado $(\leq 8 \mathrm{~mL})$ & 16 & 76,20 & 5 & 23,80 & \\
\hline Inadequado (> $8 \mathrm{~mL})$ & 211 & 83,40 & 42 & 16,60 & $0,3 / 5$ \\
\hline Consumo per capita de sal & & & & & \\
\hline Adequado $(\leq 5 \mathrm{~g})$ & 115 & 78,90 & 17 & 12,90 & סרת م \\
\hline Inadequado (>5 g) & 112 & 87,10 & 30 & 21,10 & 0,079 \\
\hline Consumo per capita de açúcar & & & & & \\
\hline Adequado $(\leq 28 \mathrm{~g})$ & 69 & 14,00 & 14 & 16,90 & 1000 \\
\hline Inadequado (>28 g) & 158 & 82,70 & 33 & 17,30 & 1,000 \\
\hline
\end{tabular}

* SM: Salário Minimo 
Tabela 3. Análise multivariada para fatores associados ao excesso de peso, com suas respectivas razões de prevalência e intervalo de confiança de 95\%, Belo Horizonte - MG, 2013.

\begin{tabular}{|c|c|c|c|}
\hline \multirow{2}{*}{ VARIÁVEIS } & \multicolumn{3}{|c|}{ EXCESSO DE PESO } \\
\hline & RP* & IC95\% & Valor de $p$ \\
\hline \multicolumn{4}{|l|}{ Colesterol alto } \\
\hline Não & 1,00 & - & \\
\hline Sim & 1,14 & $(1,04-1,26)$ & 0,004 \\
\hline \multicolumn{4}{|l|}{ Triglicerides Alto } \\
\hline Não & 1,00 & - & \\
\hline Sim & 1,11 & $(1,00-1,23)$ & 0,043 \\
\hline \multicolumn{4}{|l|}{ Medicamentos } \\
\hline Não & 1,00 & - & \\
\hline Sim & 1,19 & $(1,05-1,34)$ & 0,005 \\
\hline \multicolumn{4}{|c|}{ Prática de atividade física } \\
\hline Sim & 1,00 & - & \\
\hline Não & 0,81 & $(0,73-0,89)$ & 0,000 \\
\hline \multicolumn{4}{|l|}{ Consumo de água } \\
\hline 2 litros ou mais & 1,00 & - & \\
\hline Menos de 2 litros & 0,88 & $(0,79-0,99)$ & 0,036 \\
\hline \multicolumn{4}{|l|}{ Hábito de beliscar } \\
\hline Não & 1,00 & - & \\
\hline Sim & 1,15 & $(1,01-1,30)$ & 0,031 \\
\hline
\end{tabular}

*RP: Razão de prevalência

Ademais, o quadro de excesso de peso também foi expressivamente superior aos achados para mulheres brasileiras (POF 2002-2003, 40\% e POF 2008-2009, 48\% $)^{13-14}$ enquadrando-se como grupo alvo de ações de saúde destinadas ao controle de doenças e agravos não transmissíveis. A prevalência de obesidade também foi alarmante $(47,5 \%)$, bem superior à média nacional para mulheres $(16 \%)^{13}$.

No que tange aos aspectos socioeconômicos, cabe salientar que a falta de associação com o excesso de peso pode ser decorrente da relativa homogeneidade amostral, com maior prevalência de mulheres adultas de maior idade (>40 anos), casadas e com renda per capita de 1/2 a 1 salário mínimo. Estudo realizado por Miron e colaboradores (2009) com manipuladores de alimentos em escolas das regiões Oeste e Sul de Santa Maria, RS reafirmam essa realidade, onde as participantes tinham entre 40 e 60 anos, sendo $81,25 \%$ casadas e mais de $60,0 \%$ pertenciam a estratos econômicos mais baixos ${ }^{18}$. Bertin e colaboradores (2009) também confirmam que os manipuladores de alimentos, em geral, são oriundos dos estratos socioeconômicos menos privilegiados, especialmente no Brasil ${ }^{3}$.

Tratando-se dos aspectos de saúde, constatou-se que maior prevalência do excesso de peso entre os participantes com colesterol e triglicéride elevados, provavelmente pela ampla interface entre essas condições que se equiparam entre os mais importantes fatores de risco para doença cardiovascular aterosclerótica ${ }^{19}$. Ainda nesse cenário, o excesso de peso foi maior entre os manipuladores de alimentos que declararam uso de medicamentos, que pode se associar a intrínseca associação desta condição com outras doenças e agravos não transmissíveis (como hipertensão arterial e diabetes), que tiveram elevadas taxas na amostra em estudo e comumente demandam uso de medicamentos.

No que refere à prática de atividade física, o resultado paradoxal encontrado (maior prevalência de excesso de peso entre os participantes praticantes) pode ser explicado pela metodologia adotada que não permitiu uma análise detalhada dos dados. O instrumento utilizado não possibilitou conhecer há quanto tempo o participante havia iniciado a prática nem a qual tipo de prática se referia. Apesar da baixa plausibilidade biológica para tal associação, cabe citar a complexidade dos fatores associados ao excesso de peso. Em estudo transversal com base em dados secundários obtidos pelo VIGITEL, no ano de 2008 não foi encontrada associação entre inatividade física e excesso de peso entre as mulheres ${ }^{20}$.

Ainda sobre essa temática, observou-se uma prevalência alta de inatividade fisica $(82,8 \%)$ entre as participantes deste estudo, superior ao identificado em público similar (56,9\%). Esse dado é preocupante tendo em vista a relação desta característica como um importante fator de risco para desenvolvimento de doenças cardiovasculares e outras desordens s1-22-23. $^{2}$. 
No que concerne aos hábitos alimentares dos manipuladores de alimentos deste estudo, notou-se elevada prevalência do hábito de beliscar, superior a outro estudo com público semelhante $-35,5 \%{ }^{17}$ e associação deste com o excesso de peso. De acordo com Matos e colaboradores (2009) ${ }^{7}$, as unidades produtoras de refeições, seja coletiva ou comercial, são locais onde esses profissionais estão constantemente em contato com os alimentos e muitos os consomem durante a sua jornada de trabalho, embora não seja oficialmente permitido, o que favoreceria a prática de beliscar entre as refeições.

Outro hábito errôneo identificado no presente estudo, se refere ao consumo inadequado de água $(66,0 \%)$ que apareceu no modelo multivariado, mas cuja explicação certamente extrapola o consumo desta bebida. Sabe-se que o princípio fundamental dos programas de controle do peso corporal envolve a disponibilização do equilíbrio energético negativo, onde o consumo adequado de água é estratégico nas reduções da ingestão calórica, mediante orientações dietéticas ${ }^{24}$.

Ao analisar o consumo de frutas, açúcar e sal, verifica-se que apesar destes fatores não terem sido associados com excesso de peso, devem ser vistos com atenção já que se associam de modo inverso (frutas, legumes e verduras) ou direto (açúcares e sal) com risco de mortalidade e da ocorrência de doenças crônicas ${ }^{25}$.

Cumpre salientar, que o estudo do tipo transversal utilizado tem como limitação o fato das medições serem realizadas no mesmo período, espaço e tempo, não podendo distinguir causa-efeito do problema. Ressalta-se, entretanto, que o objetivo deste trabalho foi verificar a prevalência de excesso de peso e identificar possíveis associações com as características socioeconômicas, história de saúde e hábitos alimentares. Assim, são necessários estudos de caráter longitudinal para verificação dos fatores associados ao aumento da prevalência de excesso de peso nessa população.
Apesar das limitações, os achados ora apontados apresentam grande relevância, pois são representativos do município e denotam a importância de estratégias de promoção de saúde direcionadas a este público, que pode ser importante parceiro para ações de educação alimentar e nutricional para a comunidade escolar.

\section{CONCLUSÃO}

O estudo demonstrou que os manipuladores de alimentos de escolas públicas apresentaram um estado nutricional alarmante com elevada prevalência de excesso de peso e perfil de saúde desfavorável, que os colocam em maior risco para doenças e agravos não transmíssiveis. Adicionalmente, identificou-se que o excesso de peso foi mais prevalente entre os participantes com hipercolesterolemia, hipertrigliceridemia, uso de medicamentos e hábito de beliscar alimentos entre as refeições, contrastando com a menor prevalência entre aqueles com consumo inadequado de água e inativos fisicamente.

Esses achados podem contribuir para o meIhor entendimento sobre a saúde dos participantes e os fatores que se associam ao excesso de peso neste grupo, oportunizando o planejamento de ações direcionadas à promoção de saúde nas instituições e valorização dos manipuladores de alimentos neste processo. Esses podem ser agentes de educação alimentar e nutricional desde o momento da produção das refeições (ao manter frutas e hortaliças na preparação, sem alteração de cardápios; e reduzir adição de açúcar, por exemplo) até a distribuição (ao incentivar o consumo de frutas e hortaliças, pelos escolares).

Por fim, destaca-se a escassez de estudos sobre a saúde de manipuladores de alimentos de escolas, públicas e privadas que compromete os processos e ações para melhorias das praticas de saúde dessa população e para o comer saudável nas escolas, refletindo a emergência de novos estudos acerca desta temática.

\section{REFERÊNCIAS}

1. Organização Mundial da Saúde. Doenças de origem alimentar: enfoque para educação e saúde. São Paulo: Roca; 2006.

2. Food and Agriculture Organization. Codex Alimentarius. Recommended international codeof practice-general principles of food hygiene. CAC/RCP 1-1969, Rev. 4-2003. Roma: FAO; 2003. 
3. Bertin CHFP; Morais TB; Sigulem DM; Rezende, MA. O trabalho sob a ótica das manipuladoras de alimentos de uma unidade hospitalar. Rev. Nutr. [online]. 2009, vol.22, n.5 [acesso 2013-06-15], pp. 643-652. Disponível em: http://www. scielo.br/scielo.php?script=sci_arttext\&pid=S1415-52732009000500005\&lng=en\&nrm=iso\&tlng=pt

4. Matos, $\mathrm{CH}$, Proenca, RPC. Condições de trabalho e estado nutricional de operadores do setor de alimentação coletiva: um estudo de caso. Rev. Nutr. 2003, vol.16, n.4 pp. 493-502.

5. Carvalho AT, Muniz VM, Gomes JF, Samico I. Programa de alimentação escolar no município de João Pessoa - PB, Brasil: as merendeiras em foco. Interface (Botucatu) [serial on the Internet]. 2008 Dec [cited 2014 Feb 06]; 12 (27): 823-834. Available from: http://www.scielo.br/scielo.php?script=sci_arttext\&pid=S1414-32832008000400012\&lng=en. http://dx.doi. org/10.1590/S1414-32832008000400012.

6. Jodelet DP. Papers on Social Representations. Peer Reviewed Online Journal 2010, volume 19, 17.1-17.9. Disponível em: http://www.psych.Ise.ac.uk/psr/PSR2010/19_17Jodelet.pdf. Acesso em 20 nov. 2011.

7. Matos $\mathrm{CH}$; Proença RPC; Costa SP. Trabajo em producción de comidas: consecuencias em La alimentación y estado nutricional de los trabajadores. Med. segur. trab. [Internet]. 2009 Mar [acessado 2013-06-15]; 55(214): 91-100. Disponivel em: http://scielo.isciii.es/scielo.php?pid=S0465-546X2009000100008\&script=sci_arttext\&tlng=en

8. Scarparo ALS, Amaro FS, Oliveira AB. Caracterização e avaliação antropométrica dos trabalhadores dos restaurantes universitários da universidade federal do Rio Grande do Sul. Rev HCPA 2010;30(3):247-251

9. Browner WS, Newman TB, Cummings SR, Hulley SB. Estimando o tamanho de amostra e o poder estatístico: pontos básicos. In: Hulley SB, Cummings SR, Browner WS, Grady D, Hearst N, Newman TB, editors. Delineando a pesquisa clínica, uma abordagem epidemiológica. 2. ${ }^{\text {a }}$ ed. Porto Alegre: Artmed; 2003, 83-104.

10. World Health Organization. Obesity: Preventing and Managing the Global Epidemic. Report. Geneva; 1998.

11. Lipschitz DA. Screening for nutritional status in the elderly. Primare Care, 1994, 21(1):55-67.

12. Lopes ALC; Ferreira, AD; Santos LC. Atendimento Nutricional na Atenção Primária à Saúde: Proposição de Protocolos. Nutrição em Pauta, 2010, v. 18, p. 40-44.

13. Brasil. Fundação IBGE (Instituto Brasileiro de Geografia e Estatística). Ministério da Saúde. Pesquisa de Orçamentos Familiares - POF 2008-2009: Antropometria de crianças, adolescentes e adultos no Brasil. Rio de Janeiro: IBGE; 2010.130 p. 14. Brasil. Fundação IBGE (Instituto Brasileiro de Geografia e Estatística). Ministério da Saúde. Pesquisa de Orçamentos Familiares - POF 2002-2003: Analise da disponibilidade domiciliar de alimentos e do Estado nutricional do Brasil. Rio de Janeiro: IBGE; 2004. 43p.

15. IOM - Institute of Medicine. Dietary Reference Intakes: The Essential Guide to Nutrient Requirements. Washington (DC), 2006, 560 p.

16. Brasil; Brasil. Guia alimentar para a população brasileira: promovendo a alimentação saudável. Brasília: Ministério da Saúde, 2008, p.19-21.

17. Boclin KLS, Blank N. Excesso de peso: característica dos trabalhadores de cozinhas coletivas?. Revista Brasileira de Saúde Ocupacional, São Paulo, 2006, 31 (113): 41-47.

18. Miron VR, Stefanello CL, Mattos KM, Colomé JS, Costenaro R, Carpes AD. Profissão merendeira: perfil profissionale condições socioeconômicas. Disciplinarum Scientia. Série: Ciências da Saúde, Santa Maria,2009, v. 10, n. 1, p. 87-95.

19. Santos RD, Maranhão RC, Luz PL, Lima JC, Filho WS, Avezum A, et al. III Diretrizes brasileiras sobre dislipidemias e diretrizes de prevenção da aterosclerose do Departamento de Aterosclerose da Sociedade Brasileira de Cardiologia. Arq Bras Cardiol 2001;77(Suppl 3):1-191

20. Iser BPM, Claro RM, Catarina M, Malta DC, Neto Otaliba LM. Fatores de risco e proteção para doenças crônicas não transmissíveis obtidos por inquérito telefônico - Vigitel Brasil - 2009. Rev Bras Epidemiol 2011; 14(1) Supl.: 90-102. DOI 10.1590/S1415-790X2011000500010.

21. Freitas MCS, Minayo MCS, Ramos LB, Fontes GV, Santos LA, Souza EC et al. Escola: lugar de estudar e de comer. Ciênc. saúde coletiva [serial on the Internet]. 2013 Apr [cited 2014 Feb 06]; 18 (4): 979-985. Available from: http:// www.scielo.br/scielo.php?script=sci_arttext\&pid=S1413-81232013000400010\&lng=en. http://dx.doi.org/10.1590/ S1413-81232013000400010.

22. Sartorelli DS, Franco LJ. Tendências do diabetes mellitus no Brasil: o papel da transição nutricional. Cad. Saúde Pública [Internet]. [acessado 2013 June 15].Disponível em: http://www.scielosp.org/pdf/csp/v19s1/a04v19s1.pdf

23. Matos MF, Silva NA, Pimenta AJ, Cunha AJ. Prevalência dos fatores de risco para doença cardiovascular em funcionários do centro de pesquisas da Petrobras. Arq. Bras. Cardiol. [Internet]. 2004 Jan [acessado 2013 June 15]; 82(1): 1-4. Disponível em: http://www.scielo.br/pdf/abc/v82n1/a01v82n1.pdf

24. Guedes DP. Programas de controle do peso corporal: atividade física e nutrição. R. Min. Educ. Fís., Viçosa, 2002; 10(1):64-90.

25. World Health Organization. Diet nutrition and the prevention of chronic diseases: report of a joint WHO/FAO Expert Consultation. Geneva: World Health Organization; 2002. (WHO Technical Report Series, 916).

Recebido em: 03 de fevereiro de 2015. Aprovado em: 15 de agosto de 2015. 